\title{
SHIP BOWS WITH CONTINUOUS AND SPLASHLESS FLOW ATTACHMENT
}

\author{
M. A. D. MADURASINGHE AND E. O. TUCK ${ }^{1}$
}

(Received 17 April 1985; revised 26 June 1985)

\begin{abstract}
In two-dimensional bow-like flows past a semi-infinite body, one must in general expect a free-surface discontinuity, in the form of a splash or spray jet. However, there is numerical evidence that special body shapes do exist for which this splash is absent. In this study, we first establish conditions on the geometry of the bow in order that it should be splash-free at zero gravity, by solving the mathematical problem exactly. We then obtain solutions for finite non-zero gravity, by solving a non-linear integral equation numerically. A class of splashless body geometries with a downward directed segment at the extreme of the bow, to which the free surface attaches tangentially, is demonstrated in detail.
\end{abstract}

\section{Introduction}

The free streamline problem associated with the flow at the bow of a ship was treated within the framework of steady two-dimensional potential flow by Vanden-Broeck and Tuck [7], and by Vanden-Broeck, Schwartz and Tuck [8].

These authors constructed models for near-stern and near-bow flows, emphasizing that elimination of waves from a stern flow is equivalent to elimination of splashes from a bow flow. That is, if one has been able to construct a waveless stern flow, since there is no radiation condition for that flow, it can be reversed in direction to yield a splashless bow flow. Their scheme worked very well for stern flows, but they did not succeed in finding continuous solutions without waves.

\footnotetext{
${ }^{1}$ Department of Applied Mathematics, The University of Adelaide, Adelaide, South Australia, 5000 (c) Copyright Australian Mathematical Society 1986, Serial-fee code 0334-2700/86
} 
Although the above research was restricted to bow shapes consisting of a plane lower surface and an oblique plane front, it suggested that, for a given bow shape, splash-free solutions may not exist. However the possibility still exists that, by considering more-complicated families of bow-shape geometries, one might be able to identify a special member of such a family having the splash-free property. This possibility is also strongly suggested by more recent work of the same authors [9], and by Schmidt [5]. In particular, Tuck and Vanden-Broeck [6] have demonstrated numerically the existence of such bow shapes, and further suggested that those bow flows in which the splash drag component can be eliminated are of a bulbous character.

In the present study, we establish conditions on the geometry of the bow, in order that it should be splash-free at zero gravity, i.e. for infinite Froude number. That is, we set $g=0$, and solve the corresponding mathematical problem exactly by a hodograph method. This zero-gravity solution necessarily has either infinite or (exceptionally) zero draught, and we select those special solutions that have zero draught for further attention. Our numerical evidence then indicates that wave-free stern flows, or equivalently splash-free bow flows, exist for a finite range of gravity, i.e. for $0 \leqslant g<g_{m}$, where $g_{m}$ is an upper bound which depends on the family of bow shapes considered. A numerical scheme which determines only such splashless solutions is employed to investigate the deformation of the original bow shape, as gravity increases from zero. This task is achieved by allowing the numerical scheme to adjust one of the parameters determining the geometry of the bow.

\section{Formulation}

Consider the steady two-dimensional irrotational flow of an inviscid incompressible infinitely-deep fluid past a semi-infinite body, whose bottom surface is the plane $y=-D$, for $x<0$. A finite non-planar termination to the body lies in $x>0$, and there is tangential free-surface attachment at the point where the body ends. The flow underneath the body is assumed to approach a uniform stream of unit magnitude as $x \rightarrow-\infty$. The level $y=0$ corresponds to the level of the free surface at which the velocity is equal to 1 when $x>0$. In general, we expect a mean free-surface level $y=0$ as $x \rightarrow+\infty$, and can interpret $D$ as the "draught" of the body.

Let the potential function be $\phi$, and the stream function be $\psi$. Choose $\phi=0$ at the point of detachment of the free streamline and the body, and $\psi=0$ on the free surface and on the surface of the body. Let the value of $\phi$ at $x=0, y=-D$ be -1 ; thus the body is curved only in $-1<\phi<0$. The complex velocity $u-i v$ 
is an analytic function of the complex potential $f=\phi+i \psi$, where $u, v$ are the velocity components in the $x$ and $y$ directions respectively. The function $\tau-i \theta$ defined by

$$
\frac{d f}{d z}=u-i v=\exp (\tau-i \theta)
$$

is an analytic function of $f$ in the half plane $\psi \leqslant 0$, and tends to zero as $|f| \rightarrow \infty, \psi \leqslant 0$. Therefore, on $\psi=0$, its real part is the Hilbert transform of its imaginary part, and we have

$$
\tau(\phi)=\frac{1}{\pi} \int_{-\infty}^{+\infty} \frac{\theta(\varphi)}{\varphi-\phi} d \varphi
$$

where $\tau(\phi)$ and $\theta(\phi)$ denote respectively $\tau\left(\phi, 0_{-}\right)$and $\theta\left(\phi, 0_{-}\right)$. The integral in (2.2) is to be interpreted in the Cauchy principal-value sense. The kinematic condition on the body yields

$$
\begin{aligned}
\theta=0, & \psi=0, \phi<-1, \\
\theta=\Theta(\phi), & \psi=0,-1<\phi<0 .
\end{aligned}
$$

Here the function $\Theta(\phi)$ defines the shape of the body. An important special case is a polygonal body where $\Theta(\phi)$ is a step function, as sketched in Figure 1 . Bernoulli's equation and the condition of constant pressure on the free surface yield

$$
g y+\frac{1}{2} q^{2}=\frac{1}{2}
$$

where $y$ is given by

$$
\partial y / \partial \phi=e^{-\tau} \sin \theta
$$

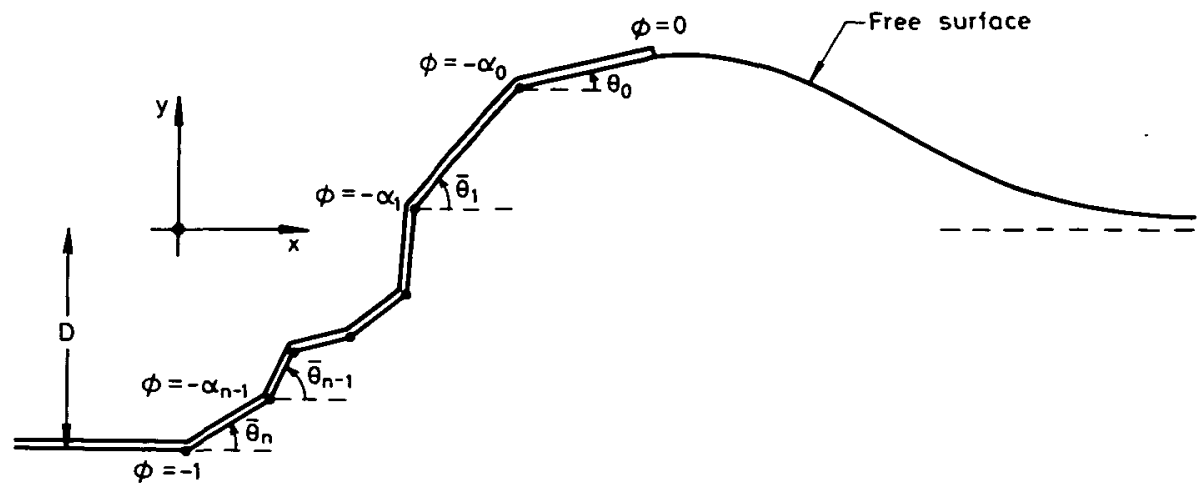

FIGURE 1. Sketch of a flow past a semi-infinite body (polygonal) with a smooth detachment. 
Differentiating (2.5) with respect to $\phi$, using (2.6), and integrating gives

$$
g \int_{\infty}^{\phi} \sin \theta d \varphi+\frac{1}{3} e^{3 \tau}=\frac{1}{3}
$$

where the limits $y \rightarrow 0$ and $\tau \rightarrow 0$ as $\phi \rightarrow \infty$ have been enforced. Substituting (2.3), (2.4) and (2.7) into (2.2), we get

$$
\int_{0}^{\infty} \frac{\theta(\varphi)}{\varphi-\phi} d \varphi+T(\phi)=\frac{\pi}{3} \log \left[1-3 g \int_{\infty}^{\phi} \sin \theta d \varphi\right] \text { for } \phi>0
$$

where

$$
T(\phi)=\int_{-1}^{0} \frac{\Theta(\varphi)}{\varphi-\phi} d \varphi
$$

$T(\phi)$ is a known function, for a given bow-shape function $\Theta(\phi)$.

For given $\Theta(\phi),-1 \leqslant \phi \leqslant 0$, the problem reduces to finding a function $\theta(\phi)$ satisfying the nonlinear integral equation (2.8) for $\phi>0$. The exact solution to equation (2.8) in the absence of gravity is described in the next section. If $g>0$, equation (2.8) must be solved numerically.

\section{Zero gravity}

As $g \rightarrow 0$, equation (2.7) indicates that $\tau \rightarrow 0$ on the free streamline; i.e. the fluid velocity is of constant (unit) magnitude. Potential flow past a curved obstacle with a constant-speed free boundary has been long recognized as an interesting mathematical problem, and questions of construction, calculation, existence, and uniqueness have intrigued many outstanding hydrodynamicists and mathematicians, see Gilbarg [2], Birkhoff \& Zarantonello [1]. For simple configurations, such as flat plates, symmetric wedges, and other polygonal shapes, the direct problem immediately admits a unique explicit solution by the hodograph method, see Wu [10], Milne-Thomson [4], Gilbarg [3].

Consider the potential function $f(z)$ given by integration of

$$
u-i v=\frac{d f}{d z}=\exp \int_{-1}^{0} \frac{\Theta^{\prime}(s)}{\pi} \log \left(\frac{\sqrt{-f}+\sqrt{-s}}{\sqrt{-f}-\sqrt{-s}}\right) d s .
$$

This could be derived as a limiting case of the Schwartz-Christoffel transformation in the hodograph plane, by allowing all the straightline segments in Figure 1 to form a smooth curve. It can be shown that (3.1) defines the complex velocity $u-i v$ as an analytic function of the complex potential $\phi+i \psi$, and satisfies the required conditions for $\psi=0$. That is

$$
u-i v \text { is real on } \psi=0, \quad \phi<-1,
$$




$$
u-i v=R(\phi) e^{-i \theta(\phi)} \quad \text { for }-1 \leqslant \phi \leqslant 0, \psi=0,
$$

for some real function $R(\phi)$,

$$
\begin{aligned}
& |u-i v|=1 \text { for } \phi>0, \psi=0, \\
& u-i v \rightarrow 1 \text { as } \phi \rightarrow \pm \infty
\end{aligned}
$$

Hence (3.1) satisfies (2.8)-(2.9) when $g=0$. Furthermore, (3.1) holds for bodies with discontinuous as well as continuous slope.

From (3.1) we can extract the free streamline slope as

$$
\theta(\phi)=\frac{1}{\pi} \int_{-1}^{0} \Theta^{\prime}(s) \arctan \left(\frac{2 \sqrt{-\phi s}}{s+\phi}\right) d s .
$$

As $\phi \rightarrow \infty, \theta(\phi)$ has the following form:

$$
\theta(\phi)=\frac{1}{\sqrt{\phi}}\left(\frac{2}{\pi} \int_{-1}^{0} \Theta^{\prime}(s) \sqrt{-s} d s\right)+O\left(\phi^{-3 / 2}\right) .
$$

The corresponding asymptotic form of the free streamline at a large distance downstream can be written as

$$
y=y_{0}+\sqrt{x}\left[\frac{2}{\pi} \int_{-1}^{0} \Theta^{\prime}(s) \sqrt{-s} d s\right]+O\left(x^{-1 / 2} \log x\right),
$$

where $y_{0}$ is a constant. The drag force $F$ is given by

$$
F=\frac{\rho}{\pi}\left[\int_{-1}^{0} \Theta^{\prime}(s) \sqrt{-s} d s\right]^{2} .
$$

where $\rho$ is the fluid density.

In general, the free streamline is parabolic at a large distance downstream, and the draught is infinite. However, there is a special case when this is not so, and it is clear that the free streamline asymptotes to a line parallel to the $x$ axis if the zero-drag condition,

$$
\int_{-1}^{0} \Theta^{\prime}(s) \sqrt{-s} d s=0
$$

is satisfied by the specified body shape. Furthermore, we can verify that $y_{0}=0$ in this $g=0$ case, by using (3.1), (3.6) and the Cauchy residue theorem in the complex plane. Thus the draught is zero in this special case; finite non-zero draught is impossible.

It should be emphasized that (3.6) is a necessary but not a sufficient condition for a physically acceptable shape of zero draught, since some profiles may cut themselves even though they satisfy (3.6). For bodies with discontinuous slopes 
(as in Figure 1), condition (3.6) should be written in the form

$$
\int_{-1}^{0} \sqrt{-s} d \Theta=0
$$

where the discontinuity at $\phi=-\alpha_{j}$ has the contribution $\sqrt{\alpha_{j}}\left(\bar{\theta}_{j+1}-\bar{\theta}_{j}\right)$. Under the zero-drag (consequently zero-draught) condition, equations (3.3) and (3.6) show that $\theta$ behaves like $\phi^{-3 / 2}$ as $\phi \rightarrow \infty$.

\section{Numerical scheme for $g \neq 0$}

To solve the integral equation (2.8) in the presence of gravity, we introduce the $N$ mesh points defining the free surface by

$$
\varphi_{i}=i^{2} \Delta^{2}, \quad i=0,1,2,3, \ldots, N-1 .
$$

The quantity $\Delta^{2}$ is a small parameter that controls the size of the intervals of discretization. Also, we introduce $N$ corresponding unknowns $\theta_{\imath}$, by the definition

$$
\theta_{i}=\theta\left(\varphi_{\imath}\right), \quad i=0,1,2, \ldots,(N-1) .
$$

The unknown $\theta_{0}$ has the special property that it is the slope of the body at $\varphi=0_{-}$as well as that of the free surface at $\varphi=0_{+}$. Thus we are assuming that the free-surface joins the body smoothly and tangentially at $\varphi=0$, and in particular are excluding the possibility that this point is a stagnation point.

We enforce the finite draught (and wave-free) requirement by assuming an asymptotic $\varphi^{-3 / 2}$ decay at infinity, by analogy with (3.3) subject to (3.6). Thus we set

$$
\theta(\varphi)=\theta_{N-1}\left[\frac{(N-1)^{2} \Delta^{2}}{\varphi}\right]^{3 / 2}, \text { for } \varphi>(N-1)^{2} \Delta^{2} .
$$

Now $\tau(\phi)$ is computed at intermediate meshpoints

$$
\phi_{i}= \begin{cases}\left(i-\frac{1}{2}\right)^{2} \Delta^{2}, & \text { if } i \neq 1 \\ \frac{1}{2} \Delta^{2}, & \text { if } i=1\end{cases}
$$

i.e. we evaluate

$$
\tau_{t}=\tau\left(\phi_{t}\right), \quad i=1,2,3, \ldots, N,
$$

in terms of $\theta_{i}$, by applying the trapezoidal rule to the integral (2.8), with the mesh points $\varphi_{1}$. The contribution to (2.8) for $\varphi>\varphi_{N-1}$ is estimated by the use of (4.3). $N$ equations are obtained from (4.5) to evaluate $\theta_{i}=\theta_{1}\left(\varphi_{1}\right), i=0,1,2, \ldots,(N-$ $1)$, for any given function $\Theta(\phi)$. 
When $g=0,(2.8)$ reduces to a system of linear equations, the solution of which agrees with the exact values to within $99 \%$ or better accuracy when $N=50$ and $\Delta^{2}=\frac{1}{600}$ and these values of $N$ and $\Delta^{2}$ were used for all calculations. Using the solution at $g \doteq 0$ as an initial guess, Newton's method seemed to converge very efficiently for a moderate value of gravity, $g=1.0$ (say). This solution is now used as an initial guess to obtain the next solution, which corresponds to a higher gravity, $g=2.0$, etc. This process of increasing gravity can be continued up to a certain upper limit $g_{m}$ (say), as discussed in the next section. The profiles of the ship bow and of the free surface were obtained by numerically integrating (2.6) and

$$
\partial x / \partial \phi=e^{-\tau} \cos \theta
$$

Some numerical results are given in Tables 1,2 and 3.

Table 1. Corresponds to Figure 2

\begin{tabular}{|l|c|c|}
\hline \multicolumn{3}{|c|}{$\alpha_{0}=.25, \bar{\theta}_{1}=\pi / 6$} \\
\hline$g$ & $\theta_{0}$ & $D$ \\
\hline 0 & -0.5300 & 0.000 \\
\hline 0.03 & -0.5120 & 0.054 \\
\hline 0.05 & -0.5004 & 0068 \\
\hline 0.07 & -0.4905 & 0.079 \\
\hline 1.0 & -0.2710 & 0.230 \\
\hline 2.0 & -0.1746 & 0.283 \\
\hline 5.0 & -0.0715 & 0.341 \\
\hline 12.0 & -0.0273 & 0.369 \\
\hline 20.0 & -0.0160 & 0.377 \\
\hline
\end{tabular}

Table 2. Corresponds to Figure 3

\begin{tabular}{|l|c|c|}
\hline \multicolumn{3}{|c|}{$\alpha_{0}=.25, \bar{\theta}_{1}=\pi / 2$} \\
\hline$g$ & $\theta_{0}$ & $D$ \\
\hline 0 & -1.590 & 0.000 \\
\hline 0.05 & -1.500 & 0.371 \\
\hline 0.09 & -1.440 & 0.481 \\
\hline 0.3 & -1.196 & 0.850 \\
\hline 1.0 & -0.555 & 0.872 \\
\hline 1.3 & -0.250 & 0.896 \\
\hline 1.4 & -0.080 & 0.930 \\
\hline 1.5 & -0.010 & 0.968 \\
\hline
\end{tabular}




\section{Discussion of results}

Consider first a polygonal body defined by

$$
\begin{aligned}
\Theta(\phi) & =\theta_{0}, & & -\alpha_{0}<\phi<0, \\
& =\bar{\theta}_{1}, & & -\alpha_{1}<\phi<-\alpha_{0}, \\
& =\bar{\theta}_{2}, & & -\alpha_{2}<\phi<-\alpha_{1}, \\
& =\cdots & & \\
& =\bar{\theta}_{n}, & & -1<\phi<-\alpha_{n-1} .
\end{aligned}
$$

Suppose first that $g=0$. Substitute into the condition (3.7) to get

$$
\bar{\theta}_{n} \sqrt{-1}+\left(\bar{\theta}_{n-1}-\bar{\theta}_{n}\right) \sqrt{\alpha_{n-1}}+\cdots+\left(\theta_{0}-\bar{\theta}_{1}\right) \sqrt{\alpha_{0}}=0
$$

which can be rewritten as

$$
-\theta_{0} \sqrt{\alpha_{0}}=\bar{\theta}_{n}\left(1-\sqrt{\alpha_{n-1}}\right)+\bar{\theta}_{n-1}\left(\sqrt{\alpha_{n-1}}-\sqrt{\alpha_{n-2}}\right)+\cdots+\bar{\theta}_{1}\left(\sqrt{\alpha_{1}}-\sqrt{\alpha_{0}}\right) .
$$

Now it is clear that, for all the body shapes which have $\bar{\theta}_{i}>0, i=1,2, \ldots, n$, the value of $\theta_{0}$ must be negative. These geometries have the property that the bow lies completely above its flat bottom level. When $g>0$ the adopted numerical procedure determines the value of $\theta_{0}$ for given $\bar{\theta}_{1}, \ldots, \bar{\theta}_{n}, \alpha_{0}, \alpha_{1}, \ldots, \alpha_{n-1}$, such that the corresponding bow flow is splashless.

On running the scheme for many different values of $\alpha_{0}, \alpha_{1}, \ldots, \alpha_{n-1}$, and $\bar{\theta}_{1}, \ldots, \bar{\theta}_{n}\left(\bar{\theta}_{i}>0\right)$, it was observed that, as $g$ increases from zero to some upper limit $g_{m}, \theta_{0}$ increases from the negative value given by (5.3) to some non-zero negative value $\theta_{m}$ (say), where $\theta_{0}<\theta_{m}<0$ for this particular class. As $g$

Table 3. Corresponds to Figure 4

\begin{tabular}{|l|c|c|}
\hline \multicolumn{3}{|c|}{$k=-25 \pi / 12$} \\
\hline$g$ & $\theta_{0}$ & $D$ \\
\hline 0 & -1.324 & 0.000 \\
\hline 0.05 & -1.284 & 0.102 \\
\hline 0.1 & -1.255 & 0.142 \\
\hline 0.2 & -1.204 & 0.194 \\
\hline 0.500 & -1.076 & 0.305 \\
\hline 1.000 & -0.890 & 0.443 \\
\hline 1.600 & -0.609 & 0.610 \\
\hline 1.694 & -0.495 & 0.664 \\
\hline
\end{tabular}


$x$

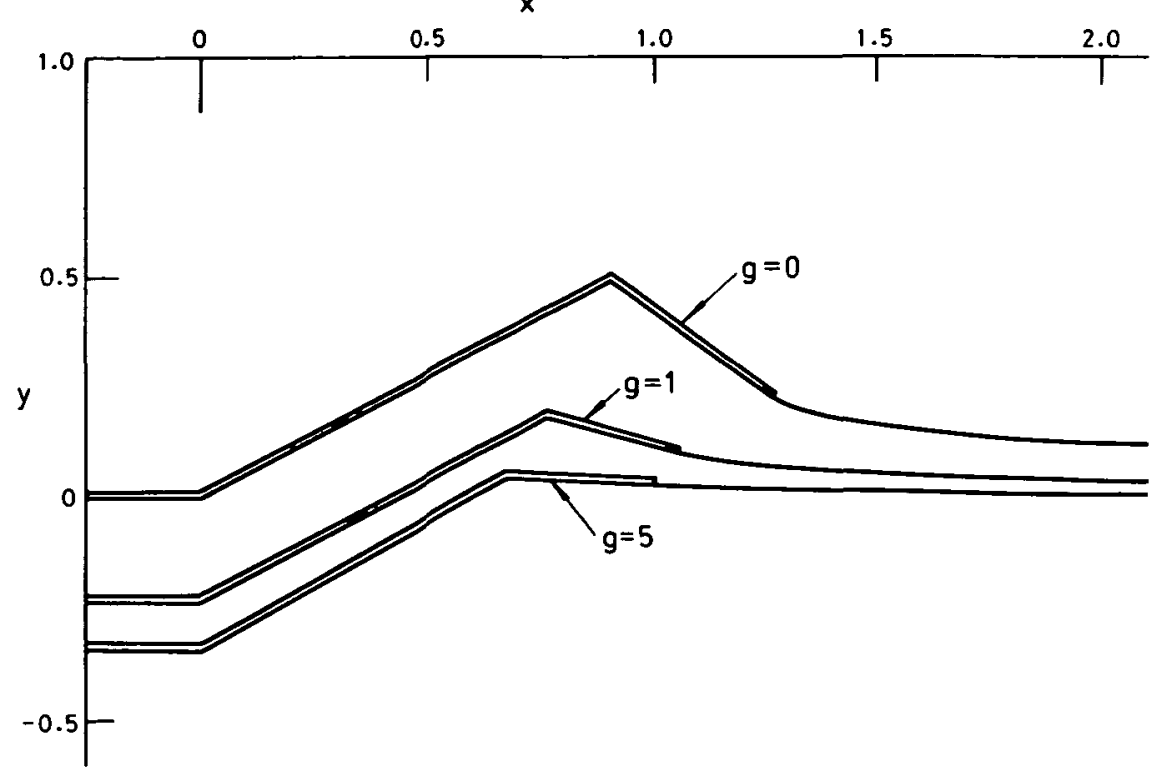

FIGURE 2. Bow with a plane oblique front and a downward angled end segment.

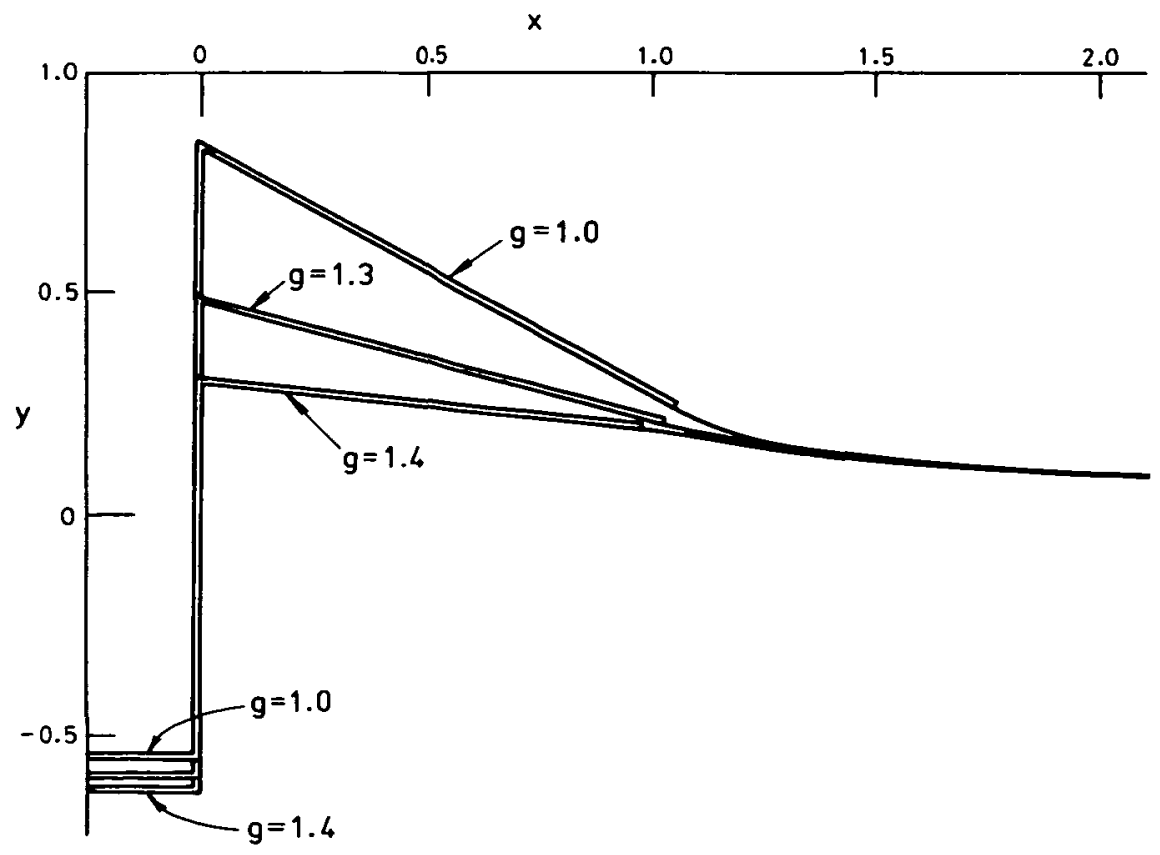

FIGURE 3. Bow with a plane vertical front and a downward angled end segment. 


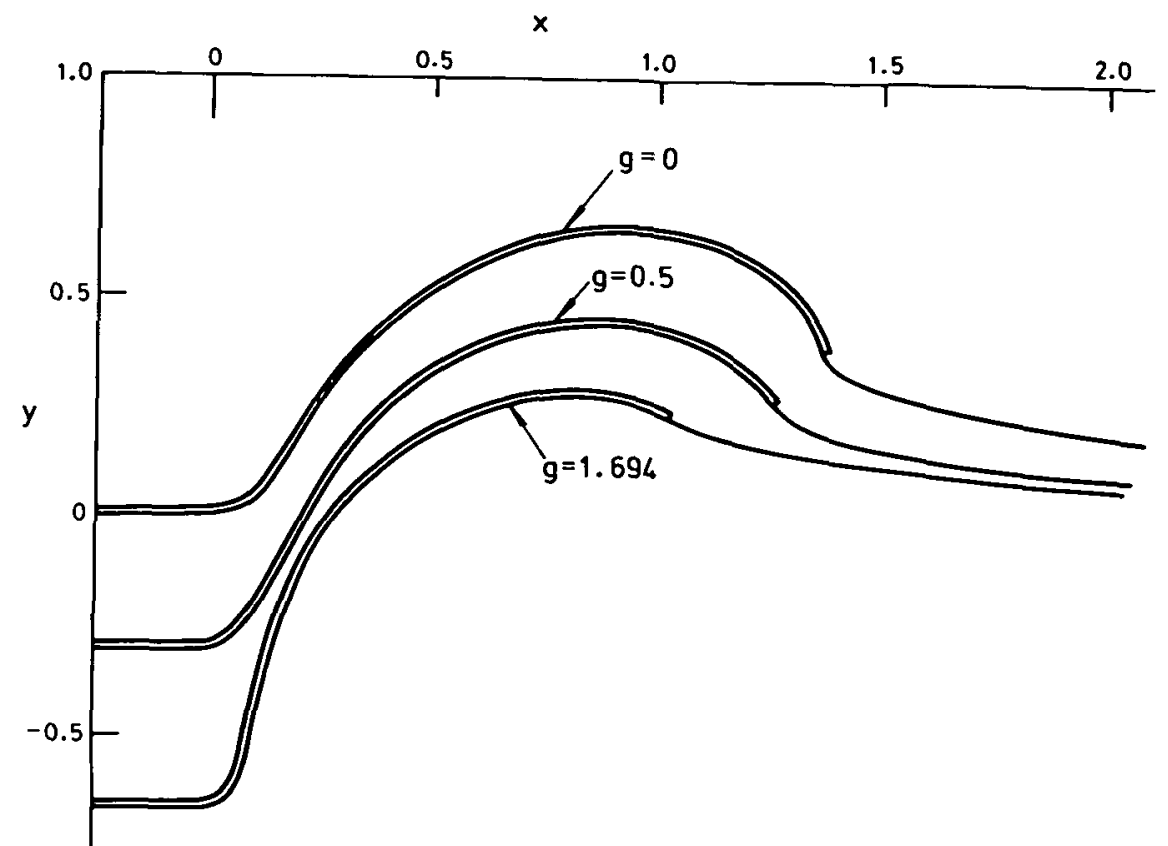

FIGURE 4. Smooth bow $\Theta(\phi)=\theta_{0}+\left(\theta_{0}+k\right) \phi+k \phi^{2}$ at $k=-25 \pi / 12$.

approached $g_{m}$, Newton's method needed more iterations to converge to a solution, and finally it failed beyond $g=g_{m}$. Figures 2 and 3 show some simple polygonal profiles of this class. The upper bound $g_{m}$ itself is not easy to estimate, but the largest values shown in Tables 1 and 2 are close to $g_{m}$.

Figure 4 corresponds to the smooth bow-shape function $\Theta(\phi)=\theta_{0}+\left(\theta_{0}+\right.$ $k) \phi+k \phi^{2}$ in the same class, where $k$ is a negative constant and $\Theta(0)=\theta_{0}$ is determined by the numerical scheme in order to yield a splashless solution for $g>0$. At $g=0$, by substituting $\Theta(\phi)$ into (3.6), we get the initial value $\boldsymbol{\theta}_{0}=k / 5$, which is negative. Further, this function has the following properties:

(i) $\Theta(\phi)>0$ for $-1<\phi<-\left(\frac{\theta_{0}+k}{2 k}\right)$,

(ii) $\Theta(\phi)<0$ for $-\left(\frac{\theta_{0}+k}{2 k}\right)<\phi<0$.

It is clear that the section (ii) with $\Theta(\phi)<0$ describes a downward-directed rudder-like segment, analogous to the last straightline segment in the case of polygonal bodies. At $k=-25 \pi / 12$, it was observed that $g_{m} \approx 1.694$ was the upper bound for splashless solutions, and $\theta_{0}$ increased from -1.32 at $g=0$ to -0.495 at $g=g_{m}$. Smaller values of $k$ produced higher values for the upper limit $g_{m}$. In Tables 1 and 2, exact upper bounds are not given, due to the fact that a very large number of computations is required to approach this number. 


\section{Concluding remarks}

The bow shapes considered in this research have the special property that they lie entirely above the plane lower surface and the flow separates tangentially from the bow. The numerical evidence strongly suggests that this class will have no splash-free solutions without a downward-angled rudder-like segment at the extreme of the bow. The possibility still exists of having a solution in which the flow rises up the front face of the body to a stagnation point, at which separation occurs. This is being explored.

Unequally spaced grid points in (4.1) and the selection of intermediate points in (4.4) increased the accuracy considerably when compared to trial runs using uniform grid. Models were tested for many different values of $N$ and $\Delta$ but $N=50$ and $\Delta=\frac{1}{600}$ gave adequate accuracy. It should be emphasized that the suggested upper bound $g_{m}$ for gravity varies according to the parameters that determine the bow geometry. In Figure 1, higher values of $g_{m}$ are obtainable by lowering the oblique flat front.

\section{References}

[1] G. Birkhoff and E. H. Zarantonello, Jets, wakes and cavittes (Academic Press, New York, 1957).

[2] D. Gilbarg, "A generalization of the Schwartz-Christoffel transformation", Proc. Nat. Acad. Sct. U.S.A. 35 (1949), 609-612.

[3] D. Gilbarg, "Jets and cavities", Handbuch der Physık, Vol. 9, 311-445 (Springer-Verlag, Berlin, 1960).

[4] L. M. Milne-Thomson, Theoretical hydrodynamics (Macmillan, London, 1968).

[5] G. H. Schmidt, "Linearized stem flow of a two-dimensional shallow-draft ship", J. Ship Res. 25 (1981), 236-242.

[6] E. O. Tuck and J.-M. Vanden-Broeck, "Splashless bow flows in two dimensions?", Proc. 15th Symp. Naval Hydro., Hamburg, Sept. 1984. pp. 293-302. (National Academy Press Washington D.C., 1985).

[7] J.-M. Vanden-Broeck and E. O. Tuck, "Computation of near-bow or stern flows, using series expansion in the Froude number", Proc. 2nd Int. Conf. Num. Ship Hydro., Berkeley (1977).

[8] J.-M. Vanden-Broeck, L. W. Schwartz, and E. O. Tuck, "Divergent low-Froude-number series expansion of nonlinear free-surface flow problems”, Proc. Roy. Soc. London. Ser. A 361(1978), 207-224.

[9] J.-M. Vanden-Broeck, and E. O. Tuck, "Wave-less free-surface pressure distributions", J. Ship Res. (1985) (in press).

[10] T. Y. Wu, “Cavity and wake flows", Ann. Rev. Fluid Mech. 4 (1972), 243-284. 